\title{
MULTI-SOURCE COOPERATIVE TRANSMISSION WITH NETWORK CODING
}

\author{
Yu-Han Yang ${ }^{\star} \quad$ Zhenzhen Gao ${ }^{\star \dagger} \quad$ K. J. Ray Liu ${ }^{\star}$ \\ *Department of ECE, University of Maryland, College Park, MD 20742, USA. \\ ${ }^{\dagger}$ Department of ICE, Xi' an Jiaotong University, Xi'an, 710049, P. R. China.
}

\begin{abstract}
A new cooperative transmission protocol with network coding scheme for synchronized multi-source system are proposed. The users are organized into two groups. The users in different groups transmit in distinct time slots, while the users in the same group transmit with orthogonal waveforms. In each transmission, the user transmits his own information and relays other users' information at the same time. The proposed protocol is able to mimic the full-duplex transmission with half-duplex mode to achieve high data rate. The network code for this protocol can be designed using classical linear block codes, whose minimum Hamming distance of the network code determines the system diversity order. Hence, with well-designed network code, the proposed protocol can achieve high diversity order. Simulation results show that the proposed scheme outperforms previous work.
\end{abstract}

\section{INTRODUCTION}

In wireless communication, channel fading presents a severe challenge for the system performance. Cooperative diversity techniques [1-3] are studied to mitigate the system outage due to channel fading. It is shown that through protocol design [1-3], user cooperation can bring the system significant diversity gains. The users help each other and form a virtual antenna array providing spatial diversity. To obtain the cooperative diversity, users transmit signals which combines different users' information. Network coding [4-6] is shown to be an effective approach to encode different information for transmission.

In a multi-source communication system, each user has his own information to transmit and also helps others' through relaying. Traditional two-stage methods $[1,7,8]$ share users' own information in the first stage and relay others' information in the second stage. Such two-stage methods can obtain high diversity order but sacrifice data rate due to the relaying stage. In [9], the two stages are combined into one by transmitting new information and relay others' information in the same stage, where time division multiple access (TDMA) is used to separate users. However, in a synchronized system, such method cannot extend to other multiple access techniques such as code division multiple access (CDMA) or frequency division multiple access (FDMA) because in the half- duplex mode, users cannot transmit and receive information at the same time. In [9], combining of different information is perfomed with the linear constellation precoding (LCP), which provides high diversity but the decoding complexity is very high.

In this paper, a new transmission protocol, which mimics the full-duplex operation via the half-duplex mode, is proposed for the synchronized multi-source system. Users transmit at the same time using orthogonal waveforms. The information combining is accomplished using network coding. We show that the system diversity order is equal to the minimum Hamming distance of the network code. We can resort to the classical linear block code to design the network code with high diversity, and the decoding is as simple as the decoding for classical linear block code.

\section{SYSTEM MODEL}

Consider a wireless multi-source cooperative communication system, where $K$ user nodes cooperate with each other to transmit information to a destination node. We assume that each node is equipped with single antenna operating in halfduplex mode, and the users are close to each other compared to their distance to the destination, so that they can receive and decode each other's information correctly.

\subsection{Cooperative Transmission Protocol}

Different from the conventional two-stage cooperative protocols, where the information of Stage 1 is relayed in Stage 2 to achieve diversity but the transmission rate is sacrificed, in the proposed protocol, new information is transmitted in each time slot and high diversity can be achieved.

The users are organized in two groups. Users in different groups transmit in distinct time slots (TDMA). Users in the same group transmit in the same time slot, but with orthogonal waveforms (e.g., CDMA or FDMA). One may argue that since the system is synchronized and orthogonal waveforms can be used, all users could transmit at the same time slot, so that only one time slot is needed. In such a way, however, all users cannot receive other user's information while they are transmitting due to the half-duplex operation. Aiming to solve 
Table 1. Proposed Cooperative Transmission Protocol

\begin{tabular}{|c|c||c|c|c|c|c|}
\hline Phase & Time slot & User 1 & User 2 & User 3 & $\ldots$ & User $K$ \\
\hline \hline \multirow{2}{*}{$n$} & $2 n$ & $\left(b_{1}(n), c_{1}(n-1)\right)$ & & & $\ldots$ & \\
\cline { 2 - 7 } & $2 n+1$ & & $\left(b_{2}(n), c_{2}(n-1)\right)$ & $\left(b_{3}(n), c_{3}(n-1)\right)$ & $\ldots$ & $\left(b_{K}(n), c_{K}(n-1)\right)$ \\
\hline \multirow{2}{*}{$n+1$} & $2 n+2$ & $\left(b_{1}(n+1), c_{1}(n)\right)$ & & & $\ldots$ & \\
\cline { 2 - 7 } & $2 n+3$ & & $\left(b_{2}(n+1), c_{2}(n)\right)$ & $\left(b_{3}(n+1), c_{3}(n)\right)$ & $\ldots$ & $\left(b_{K}(n+1), c_{K}(n)\right)$ \\
\hline
\end{tabular}

this problem, the proposed protocol mimics full-duplex operation with half-duplex by making one group listening when the other group is transmitting. When a group transmits, it not only transmits a new information bit but also relays the information bits heard from other group. These two types of information are combined in one symbol for transmission. The protocol provides high data rate since in each time slot new information bits are transmitted. High diversity can also be obtained because each user helps others through relaying.

Table 1 illustrates an example where Group 1 has only one user, User 1, and Group 2 comprises the other users. Each phase consists of two time slots. In phase $n$, User $k$ transmits $\left(b_{k}(n), c_{k}(n-1)\right)$, where $b_{k}(n)$ denotes the $n$th information bit for user $k$, and $c_{k}(n-1)$ denotes the network-coded relay bit which is generated from other users' information bits in the previous phase, i.e., $\left\{b_{k}(n-1)\right\}_{k=1}^{K}$. The two bits are together QPSK-modulated into a symbol denoted by $x_{k}(n)$. Note that in this work, we assume the users are closely located so that each user can successfully decode the signal of each other, and thus the relay bit can be formed.

We assume that the users in a group are synchronized, so that when they transmit in orthogonal waveforms, there is no inter-user interference. The destination receives $y_{k}(n)$ given by

$$
y_{k}(n)=h_{k}(n) \sqrt{P_{t}} x_{k}(n)+w_{k}(n),
$$

where $h_{k}(n)$ is an independent zero-mean complex Gaussian random variable with variance $\sigma_{h}^{2}, P_{t}$ denotes the transmit power, and $w_{k}(n)$ is AWGN with zero mean and variance $\sigma^{2}$. We assume that the channel is block fading, i.e., $h_{k}(n)$ is independent in different phases. In this paper, for the demonstration of the proposed protocol, we only consider that each user performs one transmission in each phase. In practice, in each phase a user can transmit a packet comprising a sequence of transmissions, so the assumption that $h_{k}(n)$ changes from packet to packet is more reasonable in such scenario. $h_{k}(n)$ is assumed to be known perfectly at the destination. Since each user transmits a new information bit in each phase, the data rate is high. Considering the number of orthogonal channels used, the data rate is $1 / K$ information bit per source per channel use.

The 2 bits in a QPSK symbol are lying on orthogonal bases. Thus the destination can separate the 2 bits by simply projecting the received signal onto those bases which are rotated by the channel gain $h_{k}(n)$ at the destination. The pro- jected signals are given by

$$
\begin{aligned}
& y_{k}^{b}(n)=h_{k}(n) \sqrt{\frac{P_{t}}{2}} x_{k}^{b}(n)+w_{k}^{b}(n), \\
& y_{k}^{c}(n)=h_{k}(n) \sqrt{\frac{P_{t}}{2}} x_{k}^{c}(n)+w_{k}^{c}(n),
\end{aligned}
$$

where the transmit power on each basis is $\sqrt{P_{t} / 2}$, normalized symbol power $E\left[\left(x_{k}^{b}(n)\right)^{2}\right]=E\left[\left(x_{k}^{c}(n)\right)^{2}\right]=1$ and $E\left[\left(w_{k}^{b}(n)\right)^{2}\right]=E\left[\left(w_{k}^{c}(n)\right)^{2}\right]=\sigma^{2} / 2$.

From the proposed protocol, since $\left\{c_{k}(n+1)\right\}_{k=1}^{K}$ are functions of $\left\{b_{k}(n)\right\}_{k=1}^{K}$, the collection of $\left\{y_{k}^{b}(n)\right\}_{k=1}^{K}$ and $\left\{y_{k}^{c}(n+1)\right\}_{k=1}^{K}$ is the sufficient statistic of bits $\left\{b_{k}(n)\right\}_{k=1}^{K}$. Hence, the destination can jointly decode the information bits in phase $n$ by considering the following $2 K \times 1$ vector

$$
\begin{aligned}
\mathbf{y}(n)= & {\left[y_{1}^{b}(n), y_{2}^{b}(n), \ldots, y_{K}^{b}(n),\right.} \\
& \left.y_{1}^{c}(n+1), y_{2}^{c}(n+1), \ldots, y_{K}^{c}(n+1)\right]^{T} .
\end{aligned}
$$

Each element of $\mathbf{y}(n)$ can be considered as a BPSK modulated signal.

\section{NETWORK CODING}

In this section, we characterize the design of the networkcoded relay bit and show how the code design determines the system performance. In the following, the phase index $n$ is omitted for simplicity.

Consider a $K \times K$ parity check matrix $\mathbf{P}$ with binary entries. The network-coded relay bit $c_{k}$ is given by $c_{k}=$ $\sum_{i=1}^{K} p_{i k} b_{i}$, where the operations are performed in Galois field with binary elements, i.e., the addition is a bitwise XOR operation. In matrix form, we have $\tilde{\mathbf{c}}=\mathbf{P}^{T} \mathbf{b}$, where $\tilde{\mathbf{c}}=$ $\left[c_{1}, c_{2}, \ldots, c_{K}\right]^{T}$ and $\mathbf{b}=\left[b_{1}, b_{2}, \ldots, b_{K}\right]^{T}$. Define the $1 \times 2 K$ codeword vector $\mathbf{c}=\left[\mathbf{b}^{T}, \tilde{\mathbf{c}}^{T}\right]^{T}$ and the $K \times 2 K$ generator matrix $\mathbf{G}=\left[\mathbf{I}_{K} \mid \mathbf{P}\right]$. Then the codeword $\mathbf{c}$ can be written in the systematic form,

$$
\mathbf{c}=\left[\mathbf{I}_{K} \mid \mathbf{P}\right]^{T} \mathbf{b}=\mathbf{G}^{T} \mathbf{b} .
$$

The destination can decode the information bits $\mathbf{b}$ by performing maximum likelihood (ML) decoding, which is an exhaustive search for the codeword such that its Euclidean distance to the received vector $\mathbf{y}$ is minimized.

$$
\hat{\mathbf{b}}=\arg \min _{\mathbf{b}}\left\|\mathbf{y}-\sqrt{\frac{P_{t}}{2}} \mathbf{H z}\right\|^{2},
$$


where $\mathbf{z}$ is the BPSK-modulated signal vector of a codeword c. $\mathbf{H}$ is an $2 K \times 2 K$ diagonal matrix with the corresponding channel gains of $\mathbf{y}$ as its diagonal elements, i.e., $\mathbf{H}=$ $\operatorname{diag}\left\{\left[h_{1}(n), \ldots, h_{K}(n), h_{1}(n+1), \ldots, h_{K}(n+1)\right]\right\}$ (c.f. (3)).

Since the structure of the proposed network code is simply a classical linear block code, one can easily apply classical decoding techniques to reduce the computational complexity of the ML decoding. For example, soft information of each information bit from different paths can be calculated and combined. In this paper, we use ML decoding to demonstrate the performance of the proposed protocol and network coding scheme.

\subsection{Diversity Order}

In the following we show that the minimum Hamming distance of the codewords generated by $\mathbf{G}$ determines the system diversity order. We study the system outage performance by addressing the following proposition.

Proposition 1: the diversity order is equal to $d_{\min }$, the minimum Hamming distance of the codewords generated by the generator matrix $\mathbf{G}$.

We first analyze the pairwise error probability $\operatorname{Pr}\left\{\mathbf{z}_{1} \rightarrow\right.$ $\left.\mathbf{z}_{2}\right\}$, which is the probability that the receiver decides the transmitted signal as $\mathbf{z}_{2}$ when $\mathbf{z}_{1}$ is transmitted, where $\mathbf{z}_{1}$ and $\mathbf{z}_{2}$ are BPSK-modulated signals from the codewords generated by $\mathbf{G}$. The conditional pairwise error probability [10] is given by

$$
\begin{aligned}
\operatorname{Pr}\left\{\mathbf{z}_{1} \rightarrow \mathbf{z}_{2} \mid \mathbf{H}\right\} & =Q\left(\sqrt{\frac{1}{4} \mathrm{SNR} \sum_{i=1}^{2 K}\left|h_{i}\right|^{2}\left(z_{1 i}-z_{2 i}\right)^{2}}\right) \\
& \leq Q\left(\sqrt{\operatorname{SNR} \sum_{i=1}^{d_{\min }}\left|h_{i}\right|^{2}}\right)
\end{aligned}
$$

In (6), for simplicity, we re-index the notation of $h_{i}$ to be the $i$ th diagonal component of $\mathbf{H} . z_{1 i}$ and $z_{2 i}$ denote the $i$ th elements of $\mathbf{z}_{1}$ and $\mathbf{z}_{2}$. SNR is defined as $P_{t} / \sigma^{2}$. $Q(\cdot)$ denotes the complementary cumulative distribution function of a Gaussian random variable with zero mean and unit variance. In (7), for simplicity and without loss of generality again, we assume the first $d_{\text {min }}$ elements of $\mathbf{z}_{1}$ and $\mathbf{z}_{2}$ are different.

Now taking expectation on the conditional probability with respect to $\mathbf{H}$, we obtain the pairwise codeword error probability by

$$
\operatorname{Pr}\left\{\mathbf{z}_{1} \rightarrow \mathbf{z}_{2}\right\}=E\left[\operatorname{Pr}\left\{\mathbf{z}_{1} \rightarrow \mathbf{z}_{2} \mid \mathbf{H}\right\}\right] \approx \mathcal{O}\left(\frac{1}{\mathrm{SNR}}\right)^{d_{\mathrm{min}}}
$$

At high SNR, the error probability decreases as the $d_{\min }$ th power of SNR. Therefore, the diversity order is $d_{\text {min }}$.

\subsection{Half-Duplex Constraint}

Since linear block codes have been well studied, we can choose the generator matrix $\mathbf{G}$ with larger $d_{\min }$ to obtain higher diversity. However, due to the half-duplex constraint, the design of $\mathbf{G}$ is not arbitrary. The half-duplex constraint limits the choices of feasible $\mathbf{G}$.

Specifically, let us consider in phase $(n+1)$, some user $k$ in Group 1 cannot hear from other users in the same group, so the network-coded bit $c_{k}(n)$ can only depend on $b_{k}(n)$ and the bits he received from Group 2 in phase $n$, i.e., $b_{j}(n), \forall j$ in Group 2. Similar but looser constraint exists in Group 2. Since Group 2 transmits after Group 1, Group 2 has more information than Group 1 for the construction of network-coded bit. Consider some user $j$ in Group 2. Then $c_{j}(n)$ can depend on his own bit $b_{j}(n)$, the bits received from Group 1 in phase $n$, i.e., $b_{k}(n)$, and the network-coded bits from Group 1 in phase $(n+1)$, i.e., $c_{k}(n)$, for all $k$ in Group 1 .

When $K=3$ and Group 1 has only user 1, and Group 2 has user 2 and 3 , a feasible generator matrix with $d_{\min }=3$ can be

$$
\mathbf{G}_{3}=\left[\begin{array}{lll|lll}
1 & 0 & 0 & 0 & 1 & 1 \\
0 & 1 & 0 & 1 & 0 & 1 \\
0 & 0 & 1 & 1 & 1 & 0
\end{array}\right]
$$

In phase $(n+1)$, user 1 has $b_{2}(n)$ and $b_{3}(n)$ heard in phase $n$ so he can construct the network-coded bit $c_{1}(n)=$ $b_{2}(n)+b_{3}(n)$, where the addition is in $G F(2)$. For user 2, although he cannot directly obtain $b_{3}(n)$, he can still construct the network-coded bit by $c_{2}(n)=c_{1}(n)+b_{1}(n)+b_{2}(n)=$ $b_{1}(n)+b_{3}(n)$. The case for user 3 is similar.

A feasible generator matrix with $d_{\min }=4$ for $K=4$ can be

$$
\mathbf{G}_{4}=\left[\begin{array}{llll|llll}
1 & 0 & 0 & 0 & 0 & 1 & 1 & 1 \\
0 & 1 & 0 & 0 & 1 & 0 & 1 & 1 \\
0 & 0 & 1 & 0 & 1 & 1 & 0 & 1 \\
0 & 0 & 0 & 1 & 1 & 1 & 1 & 0
\end{array}\right]
$$

We also consider user 1 in Group 1 and other users in Group 2. The feasibility of network code construction can be checked.

The proposed method can be extended in various ways. For example, we can design the network code using other codes such as convolutional codes. Note that in this work, we do not consider the inter-source error, which shall degrade the performance and thus the network code needs to be designed accordingly.

\section{SIMULATION RESULTS}

In this section, we use numerical simulation to verify the performance of the proposed protocol and network coding scheme. We assume inter-source links are perfect so each user can successfully decode other users' transmission. The focus is on the performance at the destination. 


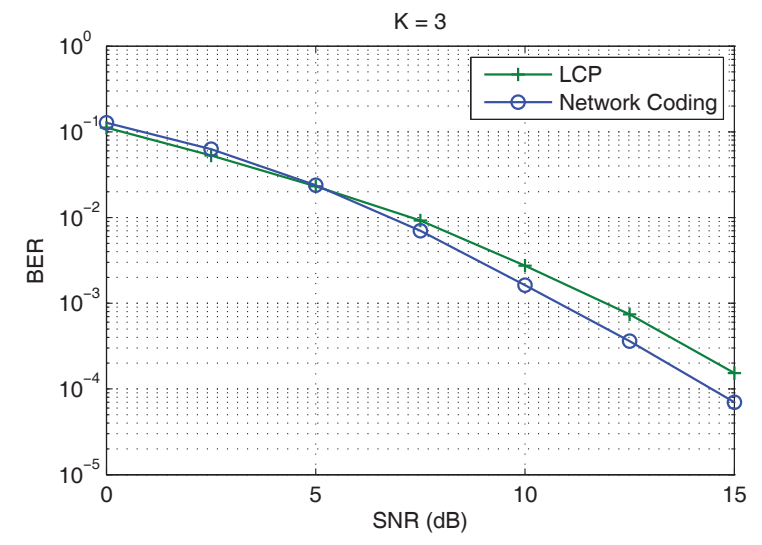

Fig. 1. BER comparison for $K=3$.

The proposed method is compared with the protocol and coding scheme in [9], where each user transmits in different time slot, so the transmission of each user can be heard by all the others. Thus the problem resulting from half-duplex does not exist in [9]. To relay the information, [9] applies linear constellation precoding (LCP), which is to linearly combine the symbols of information bits into a new symbol. An issue of such approach is its high decoding complexity if ML decoding is applied. Since all the information symbols are mixed together, joint decoding of all phases is required and results in exponentially-increasing decoding complexity with the number of phases. Instead, for the proposed scheme, even with ML decoding, the complexity is independent of the number of phases, since the information bit and the network-coded bit can be separated in two independent bases.

In our simulation, the number of phases is set as 3 for [9]. Thus their throughput is $3 /(4 K)$ information symbols per source per channel use, while the throughput of our proposed scheme is $1 / K$, which is not related to the number of phases. Only when the number of phases approaches infinity, their throughput is $1 / K$. However, smaller number of phases can be advantageous to their performance, since in the initial and final phases, smaller constellation size is used. That is, if the number of phases is set higher, the performance of [9] will not be better and the decoding complexity will be much higher.

Figure 1 and 2 show the bit error rate (BER) of a 3-user and a 4-user system, where the proposed scheme applies the generator matrices $\mathbf{G}_{3}$ in (9) and $\mathbf{G}_{4}$ in (10), respectively, as the network code for each system. In both figures, we can see that the proposed method and [9] can both achieve the same diversity, and the proposed method performs better than [9] at high SNR, where the power gain is about $1 \mathrm{~dB}$ for Figure 1 and $1.5 \mathrm{~dB}$ for Figure 2.

The performance of [9] is better at low SNR because in their method, the constellation size is smaller in the initial and the final phases. Hence, if the number of phases is small, such advantage can be prominent. On the other hand, if the

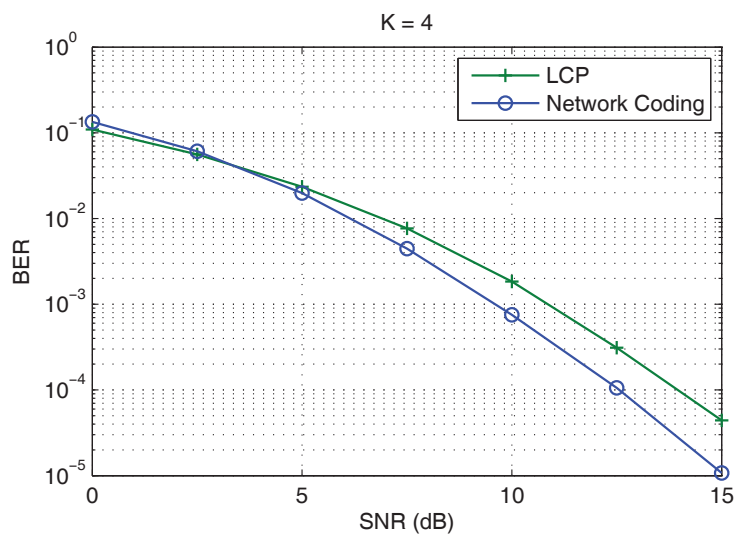

Fig. 2. BER comparison for $K=4$

number of phases is large, such advantage will be diluted.

\section{CONCLUSION}

The proposed multi-source transmission protocol mimics the full-duplex operation via the half-duplex mode and provides high data rate. The information combining is accomplished using network coding, which can be designed with classical linear block codes. We show that the system diversity order is determined by the minimum Hamming distance. With welldesigned network code, the system exhibits high diversity order and better performance compared to previous work.

\section{REFERENCES}

[1] J. N. Laneman and G. W. Wornell, "Distributed space-time-coded protocols for exploiting cooperative diversity in wireless networks," IEEE Trans. Inform. Theory, vol. 49, no. 10, pp. 2415-2425, Oct. 2003.

[2] J.N. Laneman, D.N.C. Tse, and G.W. Wornell, "Cooperative diversity in wireless networks: Efficient protocols and outage behavior," IEEE Trans. Inform. Theory, vol. 50, no. 12, Dec. 2004

[3] K. J. R. Liu, A. K. Sadek, W. Su, and A. Kwasinski, Cooperative Communications and Networking, Cambridge University Press, 2008.

[4] Lei Xiao, T. Fuja, J. Kliewer, and D. Costello, "A network coding approach to cooperative diversity," IEEE Trans. Inform. Theory, vol. 53, no. 10, pp. 3714 3722, Oct. 2007.

[5] T. Koike-Akino, P. Popovski, and V. Tarokh, "Optimized constellations for twoway wireless relaying with physical network coding," IEEE J. Select. Areas Commun., vol. 27, no. 5, pp. 773-787, June 2009.

[6] H. Q. Lai and K. J. R. Liu, "Space-time network coding," IEEE Trans. Signal Processing, vol. 59, no. 4, pp. 1706-1718, Apr. 2011.

[7] O. Shalvi, "Multiple source cooperation diversity," IEEE Commun. Lett., vol. 8, pp. $712-714$, Dec. 2004

[8] Alejandro Ribeiro, Renqiu Wang, and Georgios B. Giannakis, "Multi-source cooperation with full-diversity spectral-efficiency and controllable-complexity," IEEE J. Select. Areas Commun., vol. 25, no. 2, pp. 415-425, Feb. 2007.

[9] A. Cano, J. Gomez-Vilardebo, A.I. Perez-Neira, and G.G. Giannakis, "Highrate distributed multi-source cooperation using complex field coding," in IEEE International Conference on Acoustics, Speech and Signal Processing (ICASSP 2009), May 2009, pp. 2633-2636.

[10] D. Tse and P. Viswanath, Fundamentals of Wireless Communication, Cambridge University Press, 2005. 